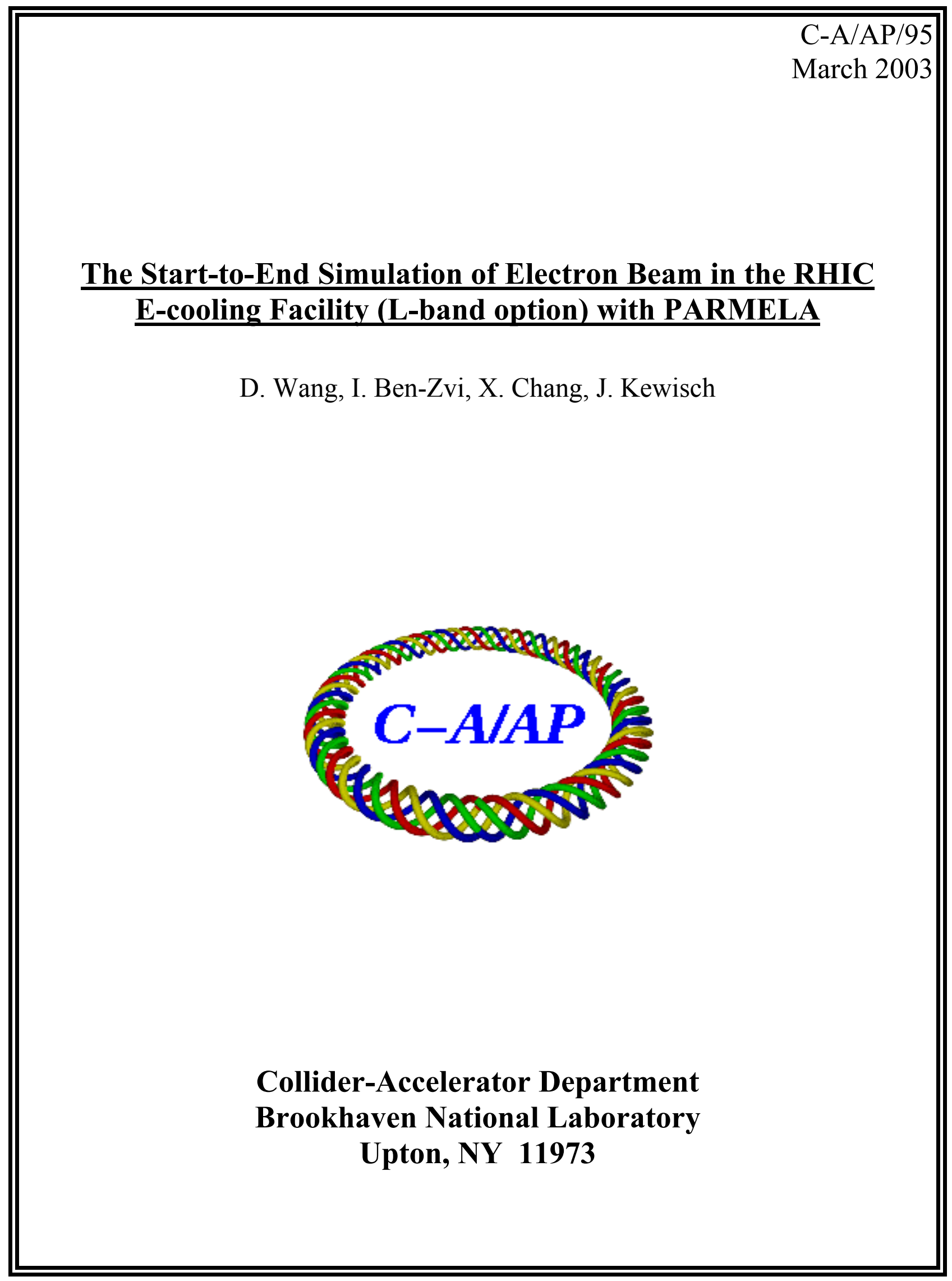




\title{
The Start-to-End Simulation of Electron Beam in the RHIC E-cooling Facility(L-band option) with PARMELA
}

\author{
Dong Wang, Ilan Ben-Zvi, Xiangyun Chang, Jorg Kewisch \\ Collider-Accelerator Department \\ Brookhaven National Laboratory, Upton, NY 11973
}

\begin{abstract}
The electron cooling facility has been proposed as a way to reduced the ion beam emittance and energy spread for the RHIC (Relativistic Heavy Ion Collider) to significantly enhance the luminosity. In a high energy electron cooling facilities like the one for RHIC, since the very higher beam quality is needed for the sufficient cooling rate, the traditional way to produce, accelerate and focus beam are hardly applicable. The new solutions include photo-injector for producing the high charge electron bunch with very low emittance, superconducting linac cavities for high current operations, strong cooler solenoid and beam transport to achieve specific beam parameters and make energyrecovery, etc. A start-to-end simulation has been performed with L-band $(1.3 \mathrm{GHz})$ gun and linac (TESLA cavity) to demonstrate the feasibility of such an unprecedented cooling facility from the beam production and transport point of view. The main results are, totally revised gun performance considering the crucial heating and RF power issues; workable scheme for merging high/low energy beam into linac with small bending angle dipole, septum magnet and rotation cavity; magnetized beam simulations, beam stretching and compressing, etc. It shows first time that such a design, with high charge beam, magnetized cathode, L-band super-conducting cavities and long transport lines, is feasible from the single bunch particle tracking point of view. The next step is to include real cooler solenoid sections (under design in the Magnet Division). The coherent synchrotron radiation effects is investigated in a separate study.
\end{abstract}




\section{Introduction}

The RHIC(Relativistic Heavy Ion Collider) is a double ring hadron collider that can provide head-on collisions at energies up to $100 \mathrm{GeV} / \mathrm{u}$ per beam for very heavy ions, normally gold $\left({ }^{197} \mathrm{Au}^{79+}\right)$ and $250 \mathrm{GeV} / \mathrm{u}$ per beam for protons. Luminosity requirements for the heaviest ions are in $10^{26} \sim 10^{27} \mathrm{~cm}^{-2} \mathrm{~s}^{-1}$ range. By now (year 2002) the achieved luminosity with $\mathrm{Au}-\mathrm{Au}$ collisions at $100 \mathrm{GeV} / \mathrm{u}$ per beam is $5 \times 10^{26} \mathrm{~cm}^{-2} \mathrm{~s}^{-1}$. In near future the RHIC luminosity can be possibly enhanced by another factor of $3 \sim 6$ with lower beta functions at IP and more bunches. The further physics goals require a higher luminosity. The limiting factor is then the large beam sizes initially determined by the injector machine and later by the Intra-Beam Scattering (IBS) effect. In order to reach that luminosity the beam sizes must be reduced by the beam cooling technique. On the other hand the concept of Electron-Ion Collider (EIC), a proposed machine complex that could provide electron-ion collision, needs even smaller beam dimensions.

Table 1, Nominal Beam Parameters for RHIC E-cooling (Ion beam) $(05 / 2002)$

\begin{tabular}{|c|c|c|}
\hline Energy & 100 & $\mathrm{GeV} /$ nucleon \\
\hline No of bunches & 120 & \\
\hline Bunch repetition rate & 9.4 & $\mathrm{MHz}$ \\
\hline Particles per bunch & $10^{9}$ & \\
\hline Revolution frequency & 78.2 & $\mathrm{kHz}$ \\
\hline Tunes & $28.2 / 29.2$ & \\
\hline Normalized emittance (95\%) (start) & 15 & pi.mm.mrad \\
\hline Bunch length & 30 & $\mathrm{~cm}$ \\
\hline Solenoid length & 30 & meter \\
\hline Solenoid field & 1.0 & Tesla \\
\hline Beta-function at cooler & 60 & meter \\
\hline Solenoid error level & $8 \times 10^{-6}$ & \\
\hline
\end{tabular}

Electron cooling has been proved to be a very effective way to reduce the beam dimensions both transversely and longitudinally in the low energy accelerators with kinetic energy less than $1 \mathrm{GeV} /$ nucluon (corresponding to $500 \mathrm{KeV}$ electron beam). In those traditional electron cooling schemes the solenoid fields accompany the electron beam from the cathode to the beam dump. While the solenoid provides the tight focusing to confine the beam the angular momentum of the beam is also kept low since the beam has never had a chance to experience the end fields of solenoid or like.

Due to the fact that the cooling rate is inversely proportional the fifth power of ion beam energy the RHIC electron cooling needs to push all the parameters to their limits to obtain the reasonable cooling time for the luminosity upgrades. Table 2 shows the parameters of electron beam according to a recent study of electron cooling simulations.[2] 
Table 2, Beam Parameters of Electron beam required by the Cooling

\begin{tabular}{|c|c|c|}
\hline Energy & 55 & $\mathrm{MeV}$ \\
\hline Particles per bunch & $3 \times 10^{10}$ & \\
\hline Charge per bunch & 10 & $\mathrm{nc}$ \\
\hline Ratio of cooler/circumference & 0.0078 & \\
\hline Average current & 47 & $\mathrm{~mA}$ \\
\hline Beta function at cooler & $\sim 5$ & $\mathrm{~meter}$ \\
\hline Transverse temperature & $\sim 330$ & $\mathrm{eV}$ \\
\hline Energy spread & $10^{-4}$ & \\
\hline Bunch length & $\sim 30$ & $\mathrm{~cm}$ \\
\hline
\end{tabular}

Among these beam parameters some are unprecedented, for example, $\sim 100 \mathrm{~mA}(\mathrm{cw})$ current for a $55 \mathrm{MeV}$ linac. The newly proven Energy Recovery Lianc(ERL) technique will be adopted to save the tremendous electric power. Still for the electron source, i.e., RF gun, which can not utilize the ER technique, is having the thermal problems due to the very high RF power therefore its peak field at cathode is limited. Consequently the beam performance in the gun is greatly deterred. From single bunch point of view, the low emittance and very small energy spread are also difficult for such a high bunch charge. On the other hand, the multi-bunch effects including Higher-Order-Modes (HOM), Beam-Break-Up(BBU) and other issues for the linac are also very tough and need through and separate investigations. Another uncertain issue is so-called magnetization of the beam. For high energy electron cooling facilities (hadron beam energy is above $10 \mathrm{GeV}$, corresponding electron beam energy is above $5 \mathrm{MeV}$ ), it is very difficult to employ a continuous solenoid field as the electron beam acceleration (especially superconducting radio frequency cavities) and the necessary long beam decompression/compression transport line can hardly be surrounded by the solenoids. The main point of transport of electron beam from the cathode to the cooler solenoid with discrete optical elements is the effect of end field of the solenoid. An analytical study was carried out [2]. Under assumptions: 1, the angular momentum dominated beam, 2, negligible energy spread, 3, linear dynamics only. It concluded that, 1, beam must be magnetized at the cathode, 2, angular momentum can be maintained through the beam line with proper matching with optical elements then eliminated while enter the cooler solenoid. Since the field strength of cooler solenoid required by the RHIC electron cooling is very strong, a rigorous simulation is vital, especially in the sense of addressing the issues the existing theory does not cover.

\section{1, General layout}

The electron beam facility consists of following major components:

1, photo-cathode RF gun and a bunching cavity

2 , beam merging system

3, superconducting linac including focusing coils

4 , transport line 1: beam stretcher including rotation cavity 
5 , cooler solenoid(under design)

6 , transport line 2: beam compressor including rotation cavity

7 , beam dump

Plot 1 shows a schematic layout of RHIC electron cooling facility. The design is still far from a frozen version.

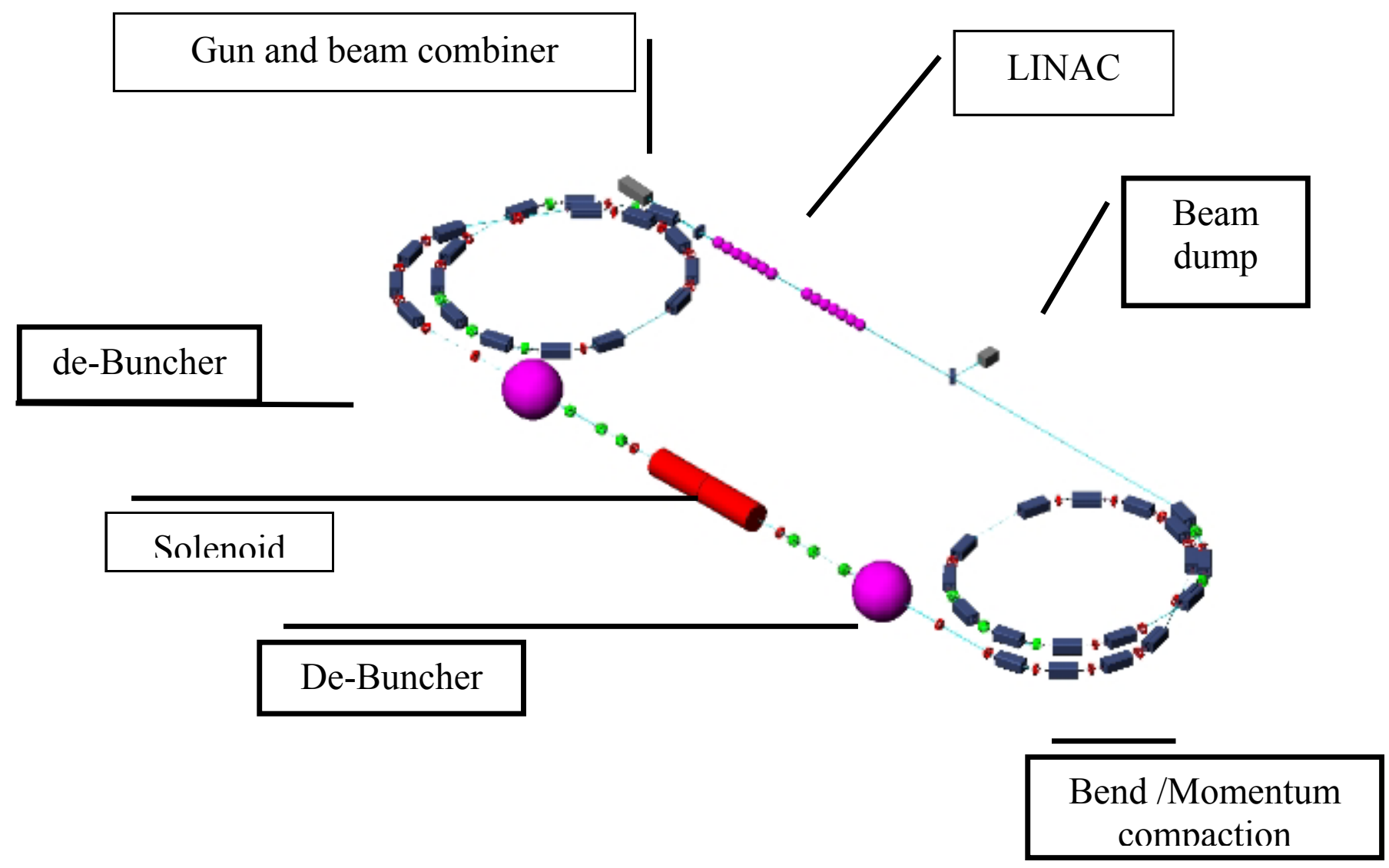

Plot 1, Sketch of electron beam facility for e-cooling

\section{2, L-band(1.3 GHz) RF Gun (photo-injector)}

In a linear accelerator the electron source plays a crucial role in determining the quality of the beam. For RHIC electron cooling facility the RF photo-injector has been chosen since it is well suitable for producing the low emittance(a few tens $\mathrm{mm}$ mrad), short(about 10ps) and intense(10nc/bunch) electron beam.

\section{1 , Choice of frequency}

At the time this study was performed, the $1.3 \mathrm{GHz}$ frequency was assumed for the gun mainly because the $1.3 \mathrm{GHz}$ TESLA 9-cell cavity was chosen as the acceleration cavity for the linac. The same frequency will simplify the system designs and operations. 


\section{2, Geometry of the gun}

A 2.5 cell structure is adopted to obtain enough energy at the exit of the gun. Plot 1 shows the gun shape and the electric field calculated by SUPERFISH.

The radius of cavity is $9.1 \mathrm{~cm}$. The iris radius is $2.75 \mathrm{~cm}$. The total length of gun is about $39 \mathrm{~cm}$. The field shown in the plot is the TE01 mode which has its highest field on the cathode. The field flatness among cells is better than 1 percent.

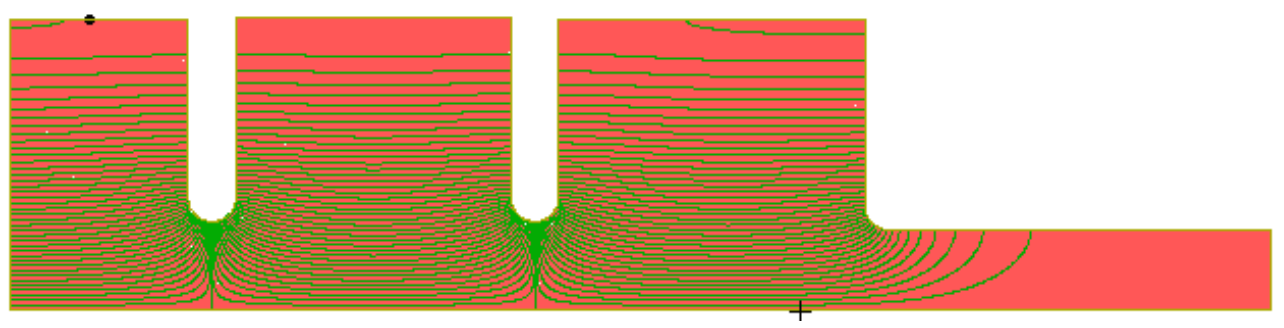

Plot 2, Geometry and Field Distribution of $1.3 \mathrm{GHz}$ RF Gun

\section{3, Determine the peak electric field in the RF gun}

The electric field in the RF gun will dictate the electron beam performance. It is one of the most important parameters in the design of RF guns. The space charge effect in an intense electron bunch can cause fast blow-up in both transverse and longitudinal dimensions. When laser incidents on the photo-cathode, the photo-electrons are produced promptly. As electrons escape from the cathode surface with quite low energies the space charge effect is particularly dangerous. The one of the main cues for this is to accelerate electrons to the high energy as quickly as possible. Therefore a high electric field gradient on the cathode is absolutely favorable.

In a previous study[3] of RF photo-injector the field gradient was set to $25 \mathrm{MV} / \mathrm{m}$ and a transverse emittance of about $10 \mathrm{~mm}$ mrad was achieved for $5 \mathrm{nc}$ bunch charge. Later on more detailed calculations in the gun thermal issues showed that the power dissipated with $25 \mathrm{MV} / \mathrm{m}$ field gradient on the cathode might be too high. Table 3 gives the results from SUPERFISH simulations.

Table 3, Major issue for a cw gun: high dissipated power

\begin{tabular}{|l|c|c|c|}
\hline Field $(\mathrm{MV} / \mathrm{m})$ & 15 & 20 & 25 \\
\hline Diss. power $(\mathrm{kw})$ & 773 & 1373 & 2140 \\
\hline Ave. power den. $\left(\mathrm{w} / \mathrm{cm}^{2}\right)$ & 293 & 520 & 810 \\
\hline Max. power den. $\left(\mathrm{w} / \mathrm{cm}^{2}\right)$ & 359 & 638 & 937 \\
\hline
\end{tabular}

$* 120^{\circ} \mathrm{C}$ operating temperature.

The preliminary thermal studies showed that the average power density should better be lower than $300 \mathrm{w} / \mathrm{cm}^{2}$. So the field gradient at the cathode is chosen to be $15 \mathrm{MV} / \mathrm{m}$. As a matter of fact, this change results in great degeneration of beam performance. 
Table 4, main parameters in optimization of gun performance

Frequency: $1.3 \mathrm{GHz}$

Number of cells: $2 \frac{1}{2}$ cell

Cathode radius: $\sim 0.9 \mathrm{~cm}$

Laser pulse length: $\sim 10$ ps

Initial RF phase: $\sim 15$ degree

Magnetic field at cathode: $0 \sim 100 \mathrm{Gs}$

Gradient at cathode: $15 \mathrm{MV} / \mathrm{m}$

Energy at exit: $\sim 2.35 \mathrm{MeV}$

External field: 3 solenoid coils

\section{4, Solenoid coils for transverse focusing and beam magnetization}

The function of solenoid coils around the gun is mainly to keep electron bunch tightly when its energy is still very low since otherwise the space charge force in an intense beam( a few nc charge/bunch with about $10 \mathrm{ps}$ length) totally destroy the electron beam before it is accelerated to high energy.

In case of RHIC electron cooler the solenoid coils have another purpose: to produce adequate magnetization for the electron beam. The detailed studies are reported in a separated paper[5]. Plot 3 shows the layout and field distribution of solenoid coils around the gun.

\section{5 , Results in RF gun simulations}

The simulations show that the overall gun performance depends on many parameters. One can hardly give the simple rule or correlations about those dependences. Basically, for a gun with high charge and low field gradient at cathode one has to enlarge the laser spot size even though it will cause increase in initial beam emittance.

Table 5, Performance of $1.3 \mathrm{G} \mathrm{Hz} \mathrm{RF} \mathrm{gun} \mathrm{with} \mathrm{zero} \mathrm{magnetic} \mathrm{field} \mathrm{at} \mathrm{cathode}$

\begin{tabular}{|l|c|c|c|}
\hline \multicolumn{1}{|c|}{ Major parameters } & Unit & $\begin{array}{c}\text { Exit of } \\
\text { RF-gun }\end{array}$ & $\begin{array}{c}\text { Entrance of } \\
\text { linac }\end{array}$ \\
\hline Beam energy & $\mathrm{MeV}$ & 2.35 & 2.35 \\
\hline Trans. emittance & $\mathrm{mm} . \mathrm{mrad}$ & 35 & 15 \\
\hline Long. emittance & $\mathrm{KeV} \cdot \mathrm{deg}$ & 32.3 & 72.1 \\
\hline Energy spread & $\%$ & 2.2 & 4.3 \\
\hline
\end{tabular}

Table 5 shows the parameters primarily optimized for the transverse emittance. Basically 15 pi mm.mrad is the lowest value one can get with the current setup. In the case of magnetized beam as the contribution of magnetization on the transverse emittance becomes dominate. When beam merging scheme is included into simulation the trade- 
off between transverse and longitudinal emittance is made to minimize the effect of the bending magnet on the beam quality.
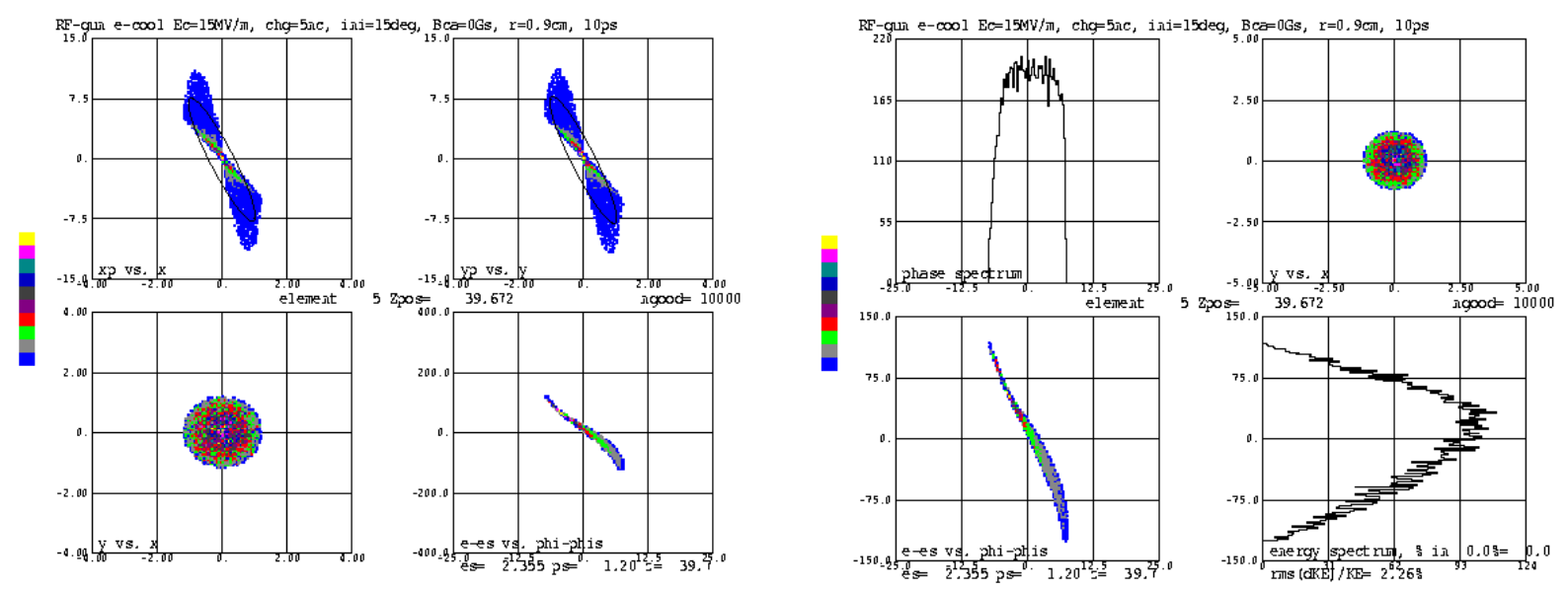

Plot 3, left: transverse phase space at gun exit, right, longitudinal phase space at gun exit.

Plot 3 shows the transverse and the longitudinal phase spaces of the beam at the gun exit.

\section{2, Magnetizing beam at cathode}

For a high energy electron cooling facility like e-cooler at the RHIC, it is very difficult to employ a continuous solenoid field as the electron beam acceleration (especially superconducting RF cavities) and the necessary long beam decompression/compression transport line can hardly be surrounded by the solenoids. The main point of transport of electron beam from the cathode to the cooler solenoid with discrete optical elements is the effect of end field of the solenoid. An analytical study was carried out [3]. Under assumptions: 1, the angular momentum dominated beam, 2, negligible energy spread, 3, linear dynamics only. It concluded that, 1 , beam must be magnetized at the cathode, 2 , angular momentum can be maintained through the beam line with proper matching with optical elements then eliminated while enter the cooler solenoid. Since the field strength of cooler solenoid required by the RHIC electron cooling is very strong, a rigorous simulation is vital, especially in the sense of addressing the issues the existing theory does not cover.

The detailed studies on the simulations of magnetized beams are summarized in another paper[5]. Here we just give a general description of magnetized beam and its application in L-band case.

If a bunch of electrons without any collective angular momentum pattern (just as most common electron beams) is simply injected into cooler solenoid, electrons will acquire some amount of angular momentum due to the transverse magnetic component in the end fields of the solenoid.

$$
M=m r^{2} \dot{\theta}
$$


Consequently the electrons get extra transverse temperatures and the angular momentum will result in modulation in transverse beam sizes in a longitudinal magnetic field. To eliminate or minimize the angular momentum the electron bunch needs to be 'magnetized', i.e., to be given certain amount of angular momentum in a specific pattern before it goes through the cooler solenoid.
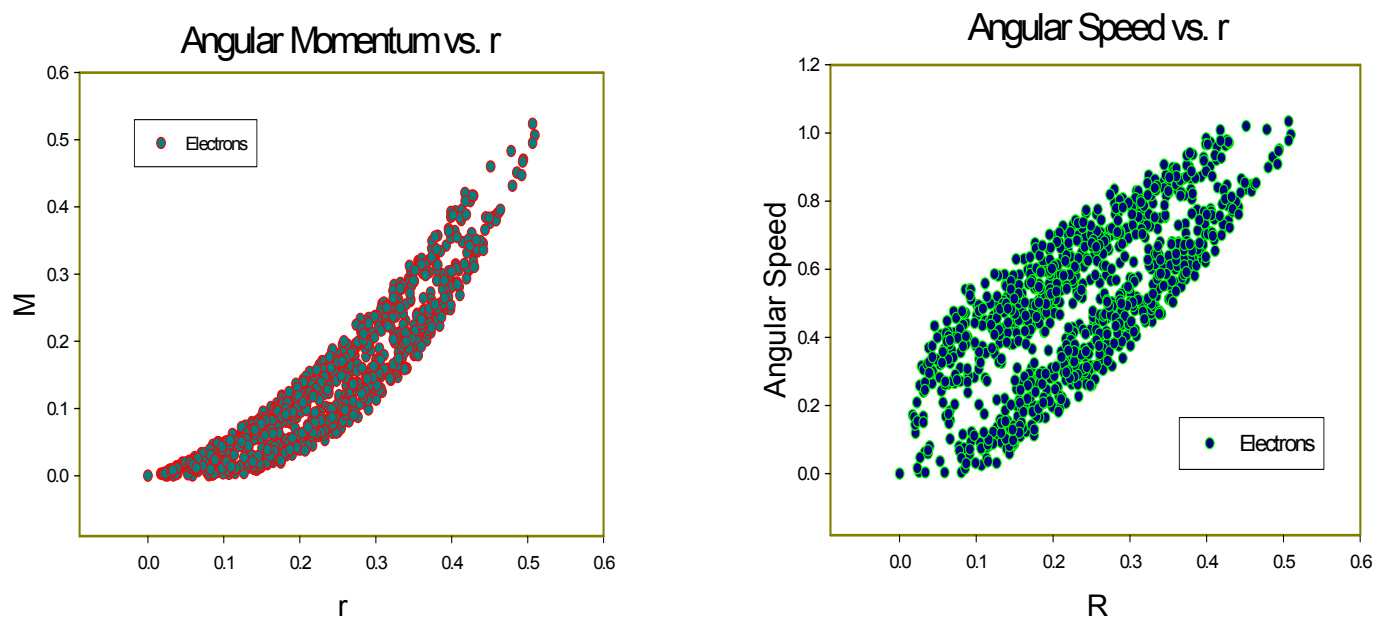

Plot 4, left, angular momentum of magnetized beam, right, angular speed

Before talking about the global matching of the magnetization of the beam, a direct consequence of a finite longitudinal magnetic field is the increase of the transverse beam emittance. The principle can be described by the Busch's theorem (which is also the simplest case of the matching of the magnetized beam),

$$
\dot{\phi}=-\frac{e}{2 \pi \gamma m_{e} r^{2}(s)}\left[\Phi(s)-\Phi_{\text {cathode }}\right]
$$

Assuming the matched electron and ion beam sizes and 1T cooler solenoid, the required field at cathode is about 100 Gausses for $10 \mathrm{~mm}$ radius beam spot size. Therefore the estimated contribution of this field is given by,

$$
\varepsilon_{\text {mag }}=\frac{\Phi}{2 \pi B \rho}
$$

The effects of this extra emittance on beam transport can only be evaluated via particle tracking. The last section will show the overall beam emittance and envelope along the beam line. 


\section{3, Low- and High-Energy beam merging system}

The principle of an Energy Recovery Linac(ERL) is to re-inject the 'used' electron beam into the same linac to give back its energy to the linear accelerator system by setting the RF phase opposite to that when it is accelerated. A beam merging system is needed to adequately guide low- and high-energy beams into the linear accelerator without damaging the bema quality.

Since there is a big difference in energy between two beams, $2 \sim 3 \mathrm{MeV}$ vs. $55 \mathrm{MeV}$, it is then possible that one could use a common bending magnet to bring two beams to the axis of the linac. The natural choice is that with a single dipole the low energy beam is bent by a large angle while the high energy beam is injected with a much smaller angle. But the simulation showed that such a big bending is quite harmful for the low energy beam. More sophisticated schemes such as achromat or other matching sections are also considered and calculated but the side effects of large bending angle dipoles can hardly overcome at this low energy and high bunch charge. Therefore a scheme was proposed that the low energy beam is bent by a minimum angle( $1 \sim 3$ degrees) while the high energy beam is brought into by a septum magnet. See plot 6 .

The criterion in design of beam merging system is like following:

$\mathrm{D}=\mathrm{D}$-low $+\mathrm{D}$-high $+\mathrm{D}$-septum $+\mathrm{M}$

D: distance between centers of high- and low-energy beam

D-low: $100 \%$ beam size (radius) of the low energy beam

D-high: $100 \%$ beam size (radius) of the high energy beam

D-septum: minimum thickness of the septum magnets

M: margin for other considerations, e.g., orbit jitter and so on.

In our case:

Table 6, Beam separation calculations

\begin{tabular}{|c|c|l|}
\hline & Amount & \multicolumn{1}{c|}{ Definition } \\
\hline D-low & $5 \mathrm{~mm}$ & $100 \%$ beam size (radius) of the low energy beam \\
\hline D-high & $3 \mathrm{~mm}$ & $100 \%$ beam size (radius) of the high energy beam \\
\hline D-septum & $10 \mathrm{~mm}$ & minimum thickness of the septum magnets \\
\hline M & $5+5 \mathrm{~mm}$ & margin for other considerations, e.g., orbit jitter and so on. \\
\hline D & $28 \mathrm{~mm}$ & distance between centers of high- and low-energy beam \\
\hline
\end{tabular}

The bending angle of the dipole and the distance between the exit of septum magnet fulfill the following relationship:

$\mathrm{D}=\mathrm{L} \times \operatorname{Sin}(\mathrm{A})$ (when $\mathrm{A}$ is small, say, a few degrees)

D: distance between centers of high- and low-energy beam, same as above definition

L: distance between the exit and the entrance of dipole

A-dipole: bending angle of the dipole before the linac.

Table 7, Different combinations of distance and bending angles $(\mathrm{D}=28 \mathrm{~mm})$

\begin{tabular}{|c|c|c|c|}
\hline L (meter) & 0.5 & 1.0 & 1.5 \\
\hline A-dipole (degree) & 3.3 & 1.6 & 1.1 \\
\hline
\end{tabular}


Table 8, Different combinations of distance and bending angles $(\mathrm{D}=35 \mathrm{~mm})$

\begin{tabular}{|c|c|c|c|}
\hline L (meter) & 0.5 & 1.0 & 1.5 \\
\hline A-dipole (degree) & 4.0 & 2.0 & 0.7 \\
\hline
\end{tabular}

In our design, the modest distance and bending angle, say, 2 degree and 1 meter, are chosen to avoid side effects on the transverse and longitudinal quality of the low energy beam.

The design of the septum magnet is preliminarily estimated by 2-D magnetic calculation code POISSON. It shows that with $10 \mathrm{~mm}$ thickness and 4000 Gausses field strength on the high energy beam side (inside septum magnet), the magnetic field leakage on the low energy beam side is negligible.

The length and bending angle of the septum magnet are determined by:

D2: total distance between high- and low energy beams,

$\mathrm{D} 2=100 \%$ beam size of high energy beam + orbit margin + high energy beam pipe

+ outer radius of solenoid coil at the end of the gun - D

D2 $=$ L-septum $\times \operatorname{Sin}($ A)

Table 9, preliminary estimation of D2

\begin{tabular}{|l|c|}
\hline The $100 \%$ beam size(radius) of high energy beam & $3 \mathrm{~mm}$ \\
\hline Orbit safe margin & $10 \mathrm{~mm}$ \\
\hline High energy beam pipe & $5 \mathrm{~mm}$ \\
\hline Outer radius of solenoid coil or bunching cavity & $\sim 90 \mathrm{~mm}$ \\
\hline D & $\sim 35 \mathrm{~mm}$ \\
\hline Total & $73 \mathrm{~mm}$ \\
\hline
\end{tabular}

* estimated as the same as the gun cavity inner radius, this is a rough estimation. Detailed design is yet to be done.

The estimated total length of septum magnet is about $30 \mathrm{~cm}$. The bending angle is about $15 \sim 20$ degrees. If the outer size of solenoid coil at the end of the gun is larger or smaller than the preliminary estimation the length or the field strength needs adjusting.

There is a bunch cavity just next to the gun exit for optimizing the longitudinal phase space of the beam and it does not provide any acceleration or de-acceleration to the center beam energy. This cavity can be the same frequency as the gun's or with higher frequency to reduce the voltage. On the other hand the optimized energy spread will help to minimize the effect of the dipole. Simulations show that, with above setup, both transverse and longitudinal parameters can fulfill the requirements even without a double dipole achromat.

A booster immediately installed after gun can significantly enhance the beam quality according to our simulation. In fact this is the way adopted in many of electron linac facilities.

\section{3, Superconducting Linac}

1.3 GHz 9-cell TESLA superconducting cavity has been a great success in recent decades in providing high gradient acceleration for the low and high energy electron linear 
accelerators. The choice of $1.3 \mathrm{GHz}$ system is mainly because it is a proven technology and a commercial product. Nevertheless the major applications of the $1.3 \mathrm{GHz} 9$-cell TESLA cavity have been in pulsed working mode. To work properly in the cw working mode in RHIC e-cooling facility some efforts need to be made to address some high current issues. Some parallel studies have been conducted to investigate the high current and multi-bunch issues[5]. From beam transport point of view, the most relevant issue is single bunch effect, i.e., RF focusing and space charge effect.

In our simulations the field gradient of 9-cell cavity is set to about 13 15 MV/m. Each 9cell cavity structure is about $1.06 \mathrm{~m}$ long. Four cavities can provide up to about $55 \mathrm{MeV}$ total energy gain. One solenoid coil in the middle of the linac is enough for the transverse focusing. Transverse emittance is maintained through the linac as expected. No third harmonic de-acceleration cavity is assumed in L-band simulation as the beam energy spread has already good enough (1.5E-3).

\section{4, De-compressor and Compressor}

The purpose of the compressor and decompressor in arcs is following:

1, optimize the energy spread before beam enters cooler solenoid to maximize the cooling rate.

2 , before beam enters the cooler solenoid, adjust(lengthen) the bunch length to partially match the ion bunch length (typically a few tens centimeters) and to mitigate the space charge effect(de-compressor). After the cooler solenoid, adjust(shorten) the bunch length to re-enter the lianc for the energy recovery(compressor).

The lattice design of the arc is done by Jorg Kewisch. The optics of compressor and decompressor is same. The bunch lengthening parameter $\mathrm{R}_{56}$ of each arc was matched to $100 \mathrm{~m}$ initially. Later on a more moderate value $(30 \mathrm{~m})$ was chosen to alleviate problems caused by large dispersions. Each arc is an achromat and has symmetric structure. In each end of arc there are enough quadrupole variables to match the twiss parameters to the linac and the cooler.

\section{5, Cavities for longitudinal phase rotation}

To minimized the energy spread of electron beam a widely used scheme is adopted in our beam line. The electron bunch is first lengthened, i.e., stretched in the longitudinal phase space by the arc, then rotated by a RF field to get a flattened energy spread. Several cavities with different frequencies range from $200 \mathrm{MHz}$ to $1.3 \mathrm{GHz}$ are used in our simulations. The voltage needed to right rotation is inversely proportional to the RF frequency. No significant difference is found in terms of beam performance.

\section{6, Summary: overall simulation results and matching of magnetized beam}

Simulations have shown that, 
1, Magnetized beam can be transported from the cathode to the cooler smoothly. This clears some worries of possible 'blow up' due to the magnetizations of the beam. See plot 5 of beam envelopes.

2, Beam transverse emittance and energy spread are well under control with the most concise schemes (septum magnet, single dipoe with small bending angle + bunching cavity). Plot 6 and 7 show that beam emittance and energy spread. The final energy beam spread, which is vital to the electron cooling rate, is about $8 \times 10^{-5}$, better than the expected $10^{-4}$ level.

3, Magnetization matching is first time seen through simulation. From plot 8 and 9 one can see the angular speeds in the electron bunch have same amplitude and distribution(with opposite sign which is not a problem). Some smears are also seen. The possible causes could be the space charge effects and optics matching errors, etc.

The cooler solenoid is not included in this simulation as there is no real design yet of this long solenoid while this work is performed. Certainly this solenoid is a key component that can affect electron beam quality inside the cooler in a big way. The bean transport from cooler to the lianc for the energy recovery is also done without any problems. It is not shown here as it is less challenging(beam quality is not that demanding).

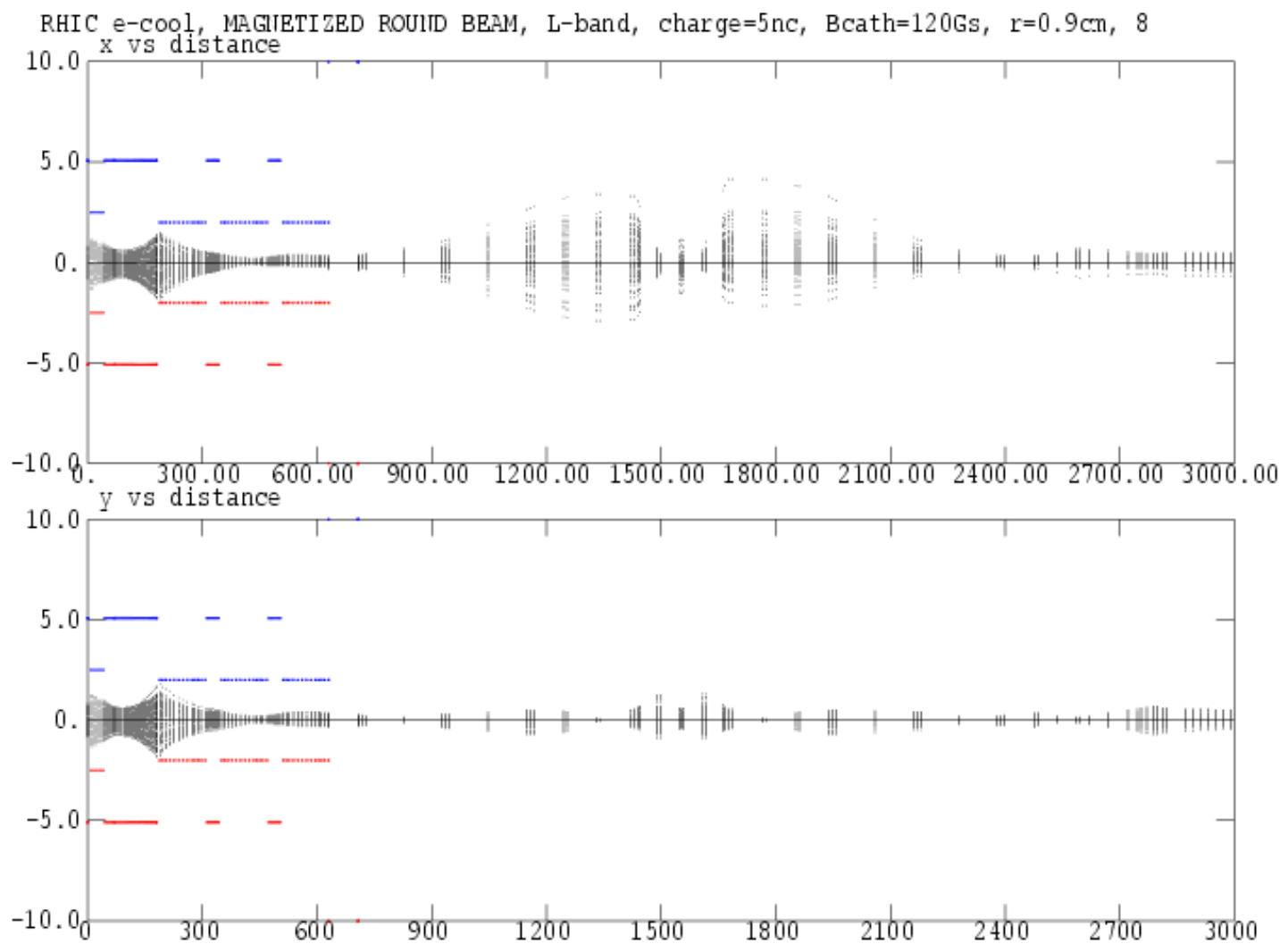

Plot 5, Beam envelopes from the cathode to the entrance of cooler 
RHIC e-cool, magnetized beam transport (photo-injector, linac, stretcher) Transverse emittance preservation

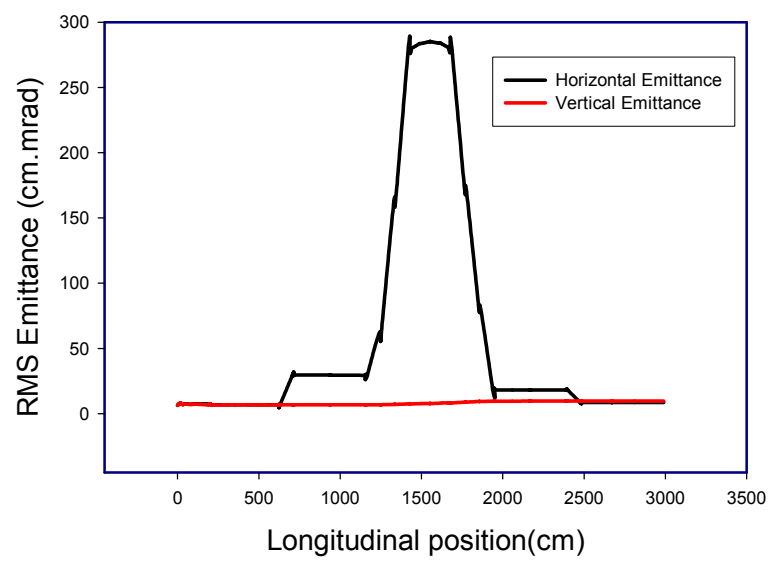

Plot 6, Hori./verti. beam emittances
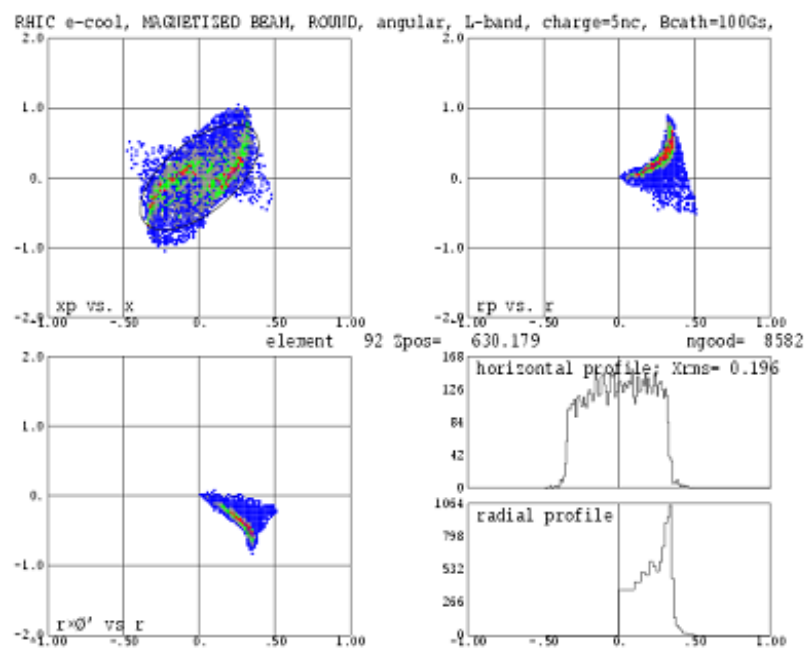

RHIC e-cool, magnetized beam transport optimization of beam energy spread

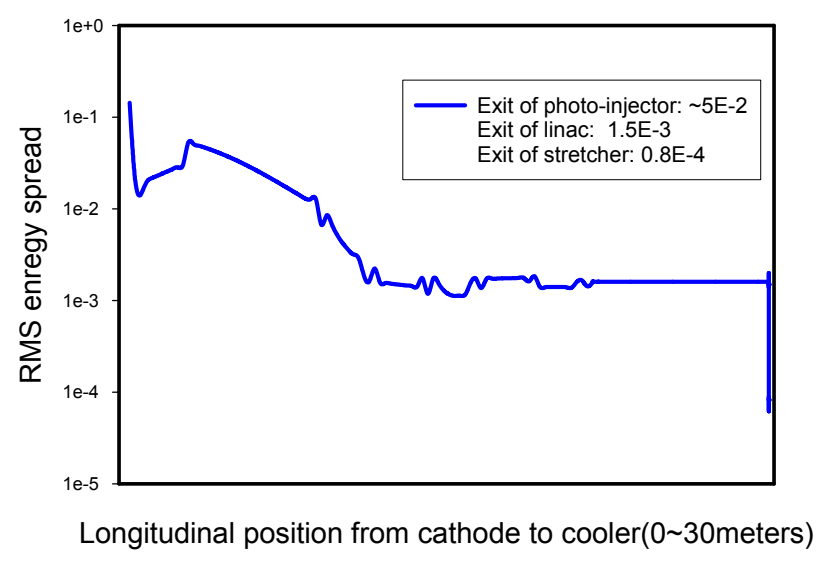

Plot 7, energy spread from cathode to cooler

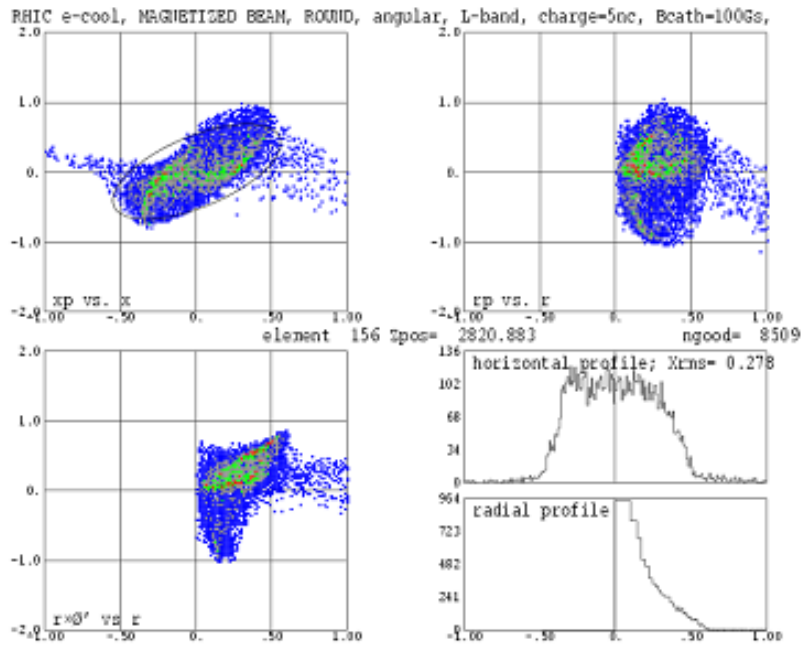

Plot 8, magnetization at linac exit

Plot 9, magnetization at entrance of the cooler 


\section{7, Higher charge(current) case}

A recent cooling simulation shows that a higher bunch charge, say, 10nc, may be needed to get decent cooling rate. This is big challenge for a L-band gun with limited field gradient at the cathode. One may have to go to lower frequency RF system which allows a bigger spot size and bunch length. However, the short wavelength of L-band system permits us to think about the bunch train scheme, i.e., using a few $5 \mathrm{nc}$ electron bunches to cool a 30 150 cm ion bunches. The bunch spacing of L-band can be as close as about 23 $\mathrm{cm}$. For a $30 \mathrm{~cm}$ ion bunch, one put 2 consecutive $5 \mathrm{nc}$ electron bunches at same time. Nevertheless high average current issues can be another limiting factor for L-band system. This needs some dedicated studies[5].

\section{8, Acknowledgment}

We would like thank L. Ahrens, M. Brennan, M. Harrison, A. Hershcovitch, D. Lowenstein, W. Mackay, Ch. Montag, S. Ozaki, S. Peggs, T. Roser, D. Trbojevic, J. Wei, and other participates of the weekly electron cooling meeting for their enlightening and stimulating discussions. We also want to think X.J. Wang, V. Yakimenko, F. Zhou for their great helps in photo-injector design and electron beam simulation issues. One of the authors (Wang) wants to thank L. Young in LANL for his tremendous help on the PARMELA code, especially for his two crucial modifications to the code (emittance calculation in different frames and illustration of angular speed) which made this work possible.

\section{Reference:}

[1] I. Ben-Zvi, et al., Electron Cooling for RHIC', PAC 2001

[2] D.Wang, I. Ben-Zvi, J. Kewisch, 'Electron Cooling for RHIC', Talk in the Electron Ion Collider Workshop 2002, Upton, NY 11973

[3] A. Burov, et al., Optical principles of beam transport for relativistic electron cooling, PRST, Accelerators and Beams, Volume 3, 094002(2000)

[4] X. Chang, et al., Simulation of 1.3GHz RF Gun, ACA Workshop, Los Angles, 2002

[5] D. Wang, et al., $700 \mathrm{MHz}$ 5-cell Superconducting RF Cavity for High Current Energy Recovery Lianc Operations, to be published

[6] D. Wang, et al., Simulations of Magnetized Beam, to appear in PAC03

[7] L. Young, private communications. 